Pacific Journal of Mathematic 


\title{
SOME EXTENSIONS OF A THEOREM OF MARCINKIEWICZ
}

\author{
Eugene LuKacs
}

1. Introduction. Let $F(x)$ be a distribution function that is a nondecreasing, right continuous function such that $F(-\infty)=0$ and $F(+\infty)=1$. The Fourier transform of $F(x)$, that is, the function

$$
f(t)=\int_{-\infty}^{\infty} e^{i t x} d F(x)
$$

is called the characteristic function of $F(x)$. It is often of interest to decide whether a given function $f(t)$ can be a characteristic function, i.e., whether it admits the representation (1.1). Necessary and sufficient conditions are known which a complex-valued function of a real variable $t$ must satisfy in order to be a characteristic function (see e. g. [7]). However, these general conditions are not easily applicable. Therefore various conditions were derived which are restricted to certain classes of functions but are applied more readily.

J. Marcinkiewicz [10] derived necessary conditions for an entire function to be a characteristic function. In the course of this study he obtained the following result:

THEOREM A. An entire function of finite order $\rho>2$ whose exponent of convergence $\rho_{1}$ is less than $\rho$ can not be a characteristic function.

As a consequence he obtained also

Theorem B. Let $P_{m}(t)$ be a polynomial of degree $m>2$ and denote by $f(t)=\exp \left[P_{m}(t)\right]$. Then $f(t)$ can not be a characteristic function.

Theorem $B$ is frequently called Marcinkiewicz' theorem. This theorem is quite often useful and was applied by many authors in studies concerning the statistical characterization of the normal distribution. A short while before the publication of Marcinkiewicz' paper G. Kunetz proved [5], [6] certain particular cases of the theorem. He did not however succeed in proving the theorem for arbitrary polynomials. Marcinkiewicz based his proof on the classical theory of entire functions. More recently D. Dugué [3] gave a new proof of Theorem B and showed that the result was due to certain convexity properties of characteristic

Received March 18, 1958. This work was supported by the National Science Foundation through grant NSF-G-4220. 
functions. He used a theorem similar to Hadamard's three circle theorem.

In the present note, Marcinkiewicz' theorem is extended to iterated exponentials and to certain functions of the form $f(t)=f_{1}(t) \exp \left[P_{m}(t)\right]$. The approach is different from the one used by either Marcinkiewicz or Dugue in so far as it is more elementary. The principal results do not require tools from the theory of entire functions but are established by means of a few results from the theory of analytic characteristic functions. In this connection it might be of interest to note that Theorem $B$ is a particular case of Theorem 1 . In this manner a rather elementary proof of Marcinkiewicz' theorem (Theorem B) is obtained which uses only the property of analytic characteristic functions which is stated as Theorem $\mathrm{D}$ in the next section. The technique can also be used to prove Theorem A; this however can not be done without using some results from the theory of entire functions.

\section{Some results from the theory of analytic characteristic functions.} A characterististic function is said to be an analytic characteristic function if it coincides with a regular analytic function in some neighborhood of the origin. The following theorem, due to R. P. Boas [1], is often useful if one wishes to show that a given characteristic function is an analytic characteristic function.

TheOREM C. Let $A(z)$ be a function of the complex variable $z$ which is regular in some neighborhood of the origin. Let $f(t)$ be a characteristic function, $\Delta>0$ a positive number and assume that $f(t)=A(t)$ if $t$ is real and if $-\Delta<t<+\Delta$. Then $f(t)$ is an analytic characteristic function.

In the following we need one of the basic properties of analytic characteristic functions which we formulate as

THEOREM D. If a characteristic function $f(z)$ is regular in a neighborhood of the origin then it is also regular in a horizontal strip of the $z$ plane and can be represented in this strip by a Fourier integral. This strip contains the origin in its interior; it may degenerate into the whole plane or into a half plane. For any horizontal line located in the interior of the strip, the modulus $|f(z)|$ attains its absolute maximum on the imaginary axis.

We say that a characteristic function is an entire characteristic function if its strip of regularity is the whole $z$-plane.

The proof of Theorem D may be found in [8] $]^{1}$ or in [9]; the second

1 Theorem 3 of $[8]$ contains an error. The statement concerning the derivatives of analytic characteristic functions is valid only for derivatives of even order. 
of these papers contains also a bibliography concerning analytic characteristic functions.

In the next section we state our results. In $\S 4$ we prove a fundamental lemma. The proofs of Theorems 1 and 2 are given in $\$ \S 5$ and 6 respectively. The final $\S 7$, contains a proof of Marcinkiewicz' Theorem A which is different from his original proof since it is based on the lemma of $\S 4$.

3. Statement of the results. We introduce first the following convenient notation for iterated exponentials.

$$
e_{1}(z)=\exp (z), e_{2}(z)=e^{e_{1}(z)}, \cdots, e_{k}(z)=e^{e_{k-1}(z)}
$$

This notation permits us to formulate our results concisely.

THEOREM 1. Let $P(t)=\sum_{v=0}^{m} c_{v} t^{v}$ be a polynomial of degree $m>2$ and for any integer $n \geqq 1$ set $f_{n}(t)=\kappa_{n} e_{n}[P(t)]$ where $\kappa_{n}^{-1}=e_{n}\left(c_{v}\right)$. Then $f_{n}(t)$ can not be a characteristic function.

The determination of the constant $\kappa_{n}$ is necessitated by the known fact that every characteristic function equals 1 for $t=0$. For $n=1$ Theorem 1 reduces to Marcinkiewicz' theorem.

Theorem 2. Let $P_{m}(t)=\sum_{v=0}^{m} c_{v} t^{v}$ be a polynomial of degree $m$. The function

$$
f(t)=\exp \left[\lambda_{1}\left(e^{i t}-1\right)+\lambda_{2}\left(e^{-i t}-1\right)+P_{m}(t)\right]
$$

is a characteristic function if and only if $\lambda_{1} \geqq 0, \lambda_{2} \geqq 0, m \leqq 2$ and $P_{2}(t)=a_{1}(i t)-a_{2} t^{2}$ where $a_{1}$ and $a_{2}$ are real and $a_{2} \geqq 0$.

This theorem contains again as a special case Marcinkiewicz' theorem; it is obtained if we put $\lambda_{1}=\lambda_{2}=0$. The proof will be based primarily on the maximum property stated in Theorem D.

4. Proof of a lemma. Before stating the lemma, we introduce the following notation which will be used consistently throughout the paper. Let $\phi(z)=\sum_{v=1}^{m} c_{v} z^{v}$ denote a polynomial of degree $m$ without constant term and with $c_{m} \neq 0$. The coefficients $c_{1}, c_{2} \cdots, c_{m}$ are arbitrary complex numbers. Define the real functions $\alpha_{1}(t, y)$ and $\beta_{1}(t, y)$ as the real and imaginary parts, respectively, of $\phi_{1}(z)$. Moreover, define

$$
A_{1}(t, y)=\alpha_{1}(t, y)-\alpha_{1}(0, y)
$$

The chief instrument in the proof of Theorem 1 is the following lemma.

Lemma 1. Let $\theta$ be an arbitrary real number. If $m>2$, there 
exists $\xi_{m} \geqq 0$ and a real number $y^{*}$ such that for $t^{*}=y^{*} \sqrt{\xi_{m}}$ and some integer $g_{1}$

$$
A_{1}\left(t^{*}, y^{*}\right)>0, \beta_{1}\left(t^{*}, y^{*}\right)-2 g_{1} \pi=\theta
$$

REMARK. The full statement of the lemma is used only in the proof of Theorem 1. The proof of Theorem 2 as well as that of Theorems $\mathrm{A}$ and $\mathrm{B}$ require only the part of the lemma which refers to the function $A_{1}\left(t^{*}, y^{*}\right)$.

Write $c_{v}=\alpha_{v}+i \beta_{v}\left(v=1,2, \cdots, m, \alpha_{v}, \beta_{v}\right.$ real $)$ and obtain for the polynomial $\phi_{1}(z)$ the expression

$$
\phi_{1}(z)=\sum_{v=1}^{m}\left(\alpha_{v}+i \beta_{v}\right)(t+i y)^{v}
$$

Set

$$
V_{s}(\xi)=\sum_{k=1}^{[s / 2]}\left(\begin{array}{l}
s \\
2 k
\end{array}\right)(-\xi)^{k-1} \text { for } s \geqq 2 \text { and } V_{1}(\xi) \equiv 0
$$

and

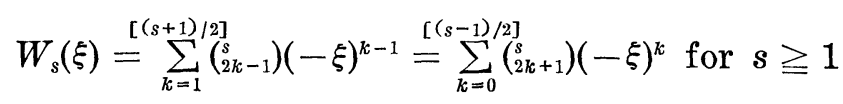

The symbol $[x]$ denotes here, as usual, the greatest integer contained in $x$. One may show by straight forward computation that

$$
(t+i y)^{2 v}=(-1)^{v} y^{2 v}\left\{1-t^{2} y^{-2} V_{2 v}\left(t^{2} y^{-2}\right)-i t y^{-1} W_{2 v}\left(t^{2} y^{-2}\right)\right\}
$$

and

$$
(t+i y)^{2 v-1}=(-1)^{v-1} y^{2 v-1}\left\{t y^{-1} W_{2 v-1}\left(t^{2} y^{-2}\right)+i\left[1-t^{2} y^{-2} V_{2 v-1}\left(t^{2} y^{-2}\right)\right]\right\}
$$

In the following we write

$$
\gamma_{2 v}=\alpha_{2 v}, \gamma_{2 v-1}=\beta_{2 v-1}, \delta_{2 v}=\beta_{2 v}, \delta_{2 v-1}=\alpha_{2 v-1}
$$

and obtain from (4.3.1), (4.3.2) and (4.4) that

$$
\begin{aligned}
& \left(\alpha_{s}+i \beta_{s}\right)(t+i y)^{s} \\
& \quad=\left\{(-1)^{[(s+1) / 2]} \gamma_{s}\left[1-t^{2} y^{-2} V_{s}\left(t^{2} y^{-2}\right)\right]+(-1)^{[s / 2]} \delta_{s} t y^{-1} W_{s}\left(t^{2} y^{-2}\right)\right\} y^{s} \\
& \quad+i\left\{(-1)^{[(s-1) / 2]} \gamma_{s} t y^{-1} W_{s}\left(t^{2} y^{-2}\right)+(-1)^{[s / 2]} \delta_{s}\left[1-t^{2} y^{-2} V_{s}\left(t^{2} y^{-2}\right)\right]\right\} y^{s}
\end{aligned}
$$

for $s=1,2, \cdots, m$.

The last formula permits the computation of $\alpha_{1}(t, y)$ and of $\beta_{1}(t, y)$; one obtains immediately

2 This is stated below as Lemma la. 
(4.6.1) $\quad \alpha_{1}(t, y)$

$$
=\sum_{s=1}^{m}\left\{(-1)^{[(s+1) / 2]} \gamma_{s}\left[1-t^{2} y^{-2} V_{s}\left(t^{2} y^{-2}\right)\right]+(-1)^{[s / 2]} \delta_{s} t y^{-1} W_{s}\left(t^{2} y^{-2}\right)\right\} y^{s}
$$

and

(4.6.2) $\quad \beta_{1}(t, y)$

$$
=\sum_{s=1}^{m}\left\{(-1)^{[(s-1) / 2]} \gamma_{s} t y^{-1} W_{s}\left(t^{2} y^{-2}\right)+(-1)^{[s / 2]} \delta_{s}\left[1-t^{2} y^{-2} V_{s}\left(t^{2} y^{-2}\right)\right]\right\} y^{s} .
$$

Since $A_{1}(t, y)=\alpha_{1}(t, y)-\alpha_{1}(0, y)$ we obtain from (4.6.1)

$$
\begin{aligned}
& A_{1}(t, y) \\
& \quad=\sum_{v=1}^{n}\left\{(-1)^{[(v-1) / 2]} \gamma_{v} t^{2} y^{-2} V_{v}\left(t^{2} y^{-2}\right)+(-1)^{[v / 2]} \delta_{v} t y^{-1} W_{v}\left(t^{2} y^{-2}\right)\right\} y^{v}
\end{aligned}
$$

we introduce a new variable $\xi=t^{2} y^{-2}(\xi \geqq 0)$ and write

$$
\begin{gathered}
A_{v}(\xi)=(-1)^{[(v-1) / 2]} \gamma_{v} \xi V_{v}(\xi)+(-1)^{[v / 2]} \delta_{v} \xi^{1 / 2} W_{v}(\xi) \\
B_{v}(\xi)=(-1)^{[(v-1) / 2]} \gamma_{v} \xi^{1 / 2} W_{v}(\xi)+(-1)^{[v / 2]} \delta_{v}\left[1-\xi V_{v}(\xi)\right],
\end{gathered}
$$

then

$$
\begin{gathered}
A_{1}(y \sqrt{\bar{\xi}}, y)=\sum_{v=1}^{m} A_{v}(\xi) y^{v} \\
\beta_{1}(y \sqrt{\xi}, y)=\sum_{v=1}^{m} B_{v}(\xi) y^{v} .
\end{gathered}
$$

The functions $A_{1}(y \sqrt{\xi}, y)$ and $\beta_{1}(y \sqrt{\xi}, y)$ are polynomials in $y$ whose coefficients depend on $\xi$. We study next the coefficients of the highest power of $y$ and prove the following two statements.

LEMMA 2. Let $m \geqq 4$, then it is possible to find a real number $\xi_{m}>0$ such that $A_{m}\left(\xi_{m}\right)>0$ while $B_{m}\left(\xi_{m}\right) \neq 0$.

Lemma 3. If $m=3$ and $\beta_{3} \neq 0$, then there exists $\xi_{3}>0$ such that $\beta_{3} A_{3}\left(\xi_{3}\right)<0$ and $B_{3}\left(\xi_{3}\right) \neq 0$. If $m=3$ and $\beta_{3}=0$ then there exists $\xi_{3}>0$ such that $A_{3}\left(\xi_{3}\right)>0$ and $B_{3}\left(\xi_{3}\right) \neq 0$.

Consider the expression $(1+i \sqrt{\xi})^{s}$ where $s$ is a positive integer and $\xi \geqq 0$ and set $\phi=\arctan \sqrt{\xi}$ with $|\phi| \leqq \frac{\pi}{2}$. Then

$$
(1+i \sqrt{\xi})^{s}=(1+\xi)^{s / 2}(\cos s \phi+i \sin s \phi) .
$$

For $s \geqq 2$ we expand $(1+i \sqrt{\xi})^{s}$ according to the binomial theorem and obtain 


$$
\begin{aligned}
(1+i \sqrt{\xi})^{s} & =\sum_{k=0}^{[s / 2]}\left(\begin{array}{l}
s \\
2 k
\end{array}\right)(-\xi)^{k}+i \sum_{k=0}^{[(s-1) / 2]}\left({ }_{2 k+1}^{s}\right)(-1)^{k} \xi^{(2 k+1) / 2} \\
& =1-\xi V_{s}(\xi)+i \sqrt{\xi} W_{s}(\xi) .
\end{aligned}
$$

We note that $(1+\xi)^{(s / 2)}=(\cos \phi)^{-s}$ and obtain from (4.9.1) and (4.9.2)

$$
\begin{gathered}
1-\xi V_{s}(\xi)=(\cos s \phi) /(\cos \phi)^{s} \\
\sqrt{\xi} W_{s}(\xi)=(\sin s \phi) /(\cos \phi)^{s} .
\end{gathered}
$$

For the sake of brevity we introduce the notation

$$
\begin{aligned}
\Gamma & =(-1)^{[(m-1) / 2]} \gamma_{m} \\
\Delta & =(-1)^{[m / 2]} \delta_{m}
\end{aligned}
$$

and express the functions $A_{m}(\xi)$ and $B_{m}(\xi)$ in terms of the variable $\phi$. If we write

$$
\xi=\tan ^{2} \phi, C(\phi)=A_{m}\left(\tan ^{2} \phi\right), D(\phi)=B_{m}\left(\tan ^{2} \phi\right)
$$

we get from (4.7), (4.10) and (4.11)

$$
\begin{gathered}
C(\phi)=I^{\prime}\left[1-\frac{\cos m \phi}{(\cos \phi)^{m}}\right]+\Delta \frac{\sin m \phi}{(\cos \phi)^{m}} \\
D(\phi)=\Gamma \frac{\sin m \phi}{(\cos \phi)^{m}}+\Delta \frac{\cos m \phi}{(\cos \phi)^{m}} .
\end{gathered}
$$

We prove Lemma 2 by showing that it is always possible to find a value $\phi_{0}$ such that $C\left(\phi_{0}\right)>0$ while $D\left(\phi_{0}\right) \neq 0$.

We give the following rule for the selection of $\phi_{0}$ :

(I) If $\Gamma>0$ and $\Delta \geqq 0$ then select $\phi_{0}$ so that $\frac{\pi}{2 m}<\phi_{0}<\frac{\pi}{m}$ while $\tan m \phi_{0} \neq-\Delta / \Gamma$

(II) If $\Gamma>0$ and $\Delta<0$ then select $\phi_{0}$ so that $\frac{\pi}{m}<\phi_{0}<\frac{5 \pi}{4 m}$ while $\tan m \phi_{0} \neq-\Delta / \Gamma$

(III) If $\Gamma=0$ and $\Delta>0$ then select $\phi_{0}$ so that $\frac{\pi}{2 m}<\phi_{0}<\frac{\pi}{m}$

(IV) If $\Gamma=0$ and $\Delta<0$ then select $\phi_{0}$ so that $\frac{\pi}{m}<\phi_{0}<\frac{5 \pi}{4 m}$

(V) If $\Gamma<0$ select a value $\phi_{0}$ which satisfies the following three conditions : 


$$
\begin{aligned}
& \text { (a) } \frac{\pi}{m}<\phi_{0}<\frac{2 \pi}{m} \\
& \text { (b) } \tan m \phi_{0} \neq-\Delta / \Gamma^{r} \\
& \text { (c) } h\left(\phi_{0}\right)=\Gamma\left(\cos ^{m} \phi_{0}-\cos m \phi_{0}\right)+\Delta \sin m \phi_{0}>0 .
\end{aligned}
$$

To show that it is possible to select $\phi_{0}$ in case (V) so that condition (c) is satisfied, we observe that $h(\phi)=\Gamma\left(\cos ^{m} \phi-\cos m \phi\right)+\Delta \sin m \phi$ is a continuous function and that $h(2 \pi / m)>0$. Hence the function $h(\phi)$ will be positive in some neighborhood of $\phi=2 \pi / m$ so that a solution in accordance with $V$ is possible. The assumption $c_{m} \neq 0$ implies that $\Gamma$ and $\Delta$ can not vanish simultaneously so that the selection covers all possibilities. Using this fact as well as the assumption $m \geqq 4$ it is easily seen that the value $\phi_{0}$ whose selection we just described satisfies the conditions $C\left(\phi_{0}\right)>0$ and $D\left(\phi_{0}\right) \neq 0$. But then it is seen from (4.12) that $\xi_{m}=\tan ^{2} \phi_{0}$ satisfies the conditions of Lemma 2 .

We prove next Lemma 3. We see from (4.7) that

$$
\begin{aligned}
& A_{3}(\xi)=-3 \beta_{3} \xi-\alpha_{3} \bigvee \bar{\xi}(3-\xi) \\
& B_{3}(\xi) \equiv-\beta_{3} \bigvee \xi(3-\xi)-\alpha_{3}(1-3 \xi) .
\end{aligned}
$$

If $\beta_{3} \neq 0$ and $\alpha_{3} \beta_{3}<0$, choose $\xi_{3}>3$ and if $\beta_{3} \neq 0, \alpha_{3} \beta_{3} \geqq 0$ choose $0<\xi_{3}<3$. If $\beta_{3}=0$ select $\xi_{3}>3$ if $\alpha_{3}>0$ but $0<\xi_{3}<3$ if $\alpha_{3}<0$.

In the following we assume that $m \geqq 3$ and choose $\xi_{m}$ in accordance with Lemmas 2 and 3 respectively. We write

$$
A_{v}=A_{v}\left(\xi_{m}\right), B_{v}=B_{v}\left(\xi_{m}\right)
$$

and from (4.7) and (4.8) obtain

$$
A_{1}\left(y \sqrt{\xi_{m}}, y\right)=A_{m} y^{m}+\sum_{v=1}^{m-1} A_{v} y^{v} .
$$

Let now $m \geqq 4$, then

$$
A_{1}\left(y \sqrt{\xi_{m}}, y\right)=A_{m} y^{m}[1+o(1)]
$$
as $y \rightarrow \infty$.

We see from Lemma 2 that $A_{m}>0$, so that $A_{1}\left(y \sqrt{\xi_{m}}, y\right)$ is positive for sufficiently large positive values of $y$.

We consider next the case $m=3$ and write $\varepsilon=\operatorname{sign} y=y /|y|$. We choose $\varepsilon$ so that $\varepsilon \beta_{3}<0$. Then

$$
A_{1}\left(y \sqrt{\xi_{3}}, y\right)=A_{3} \varepsilon|y|^{3}+A_{2} y^{2}+A_{1} \varepsilon|y|=A_{3}|y|^{3}[1+o(1)] .
$$

We know from Lemma 3 that $\varepsilon A_{3}>0$ so that $A_{1}\left(y \sqrt{\xi_{3}}, y\right)$ becomes positive if the sign of $y$ is opposite to the sign of $\beta_{3}$ and if $|y|$ is sufficiently large. We summarize these findings in the following statement: 
LEMma 1a. Let $m \geqq 3$ and suppose that one or the other of the following two conditions is satisfied.

(i) $m>3$ or $m=3$ and $\beta_{3}=0$

(ii) $m=3$ and $\beta_{3} \neq 0$.

Then there exists $a \xi_{m} \geqq 0$ and an $\tilde{A}>0$ such that

$$
A_{1}\left(y \sqrt{\xi_{m}}, y\right)=\tilde{A}|y|^{m}[1+o(1)]
$$

where the estimate holds in case (i) as $y \rightarrow \infty$ but in case (ii) as $\left(-\operatorname{sign} \beta_{3}\right) y \rightarrow \infty$. Then there exists also a value $Y=Y(m)$ such that

$$
A_{1}\left(y \sqrt{\xi_{m}}, y\right)>0
$$

provided that in case (i) $y \geqq Y$ while in case (ii) one must require $\left(-\operatorname{sign} \beta_{3}\right) y \geqq Y$.

We choose again $\xi_{m}$ in accordance with Lemmas 2 and 3 respectively and consider the polynomial

$$
B(y)=\beta_{1}\left(y \vee \xi_{m}, y\right)=\sum_{v=1}^{m} B_{v} y^{v} .
$$

Here $B_{v}$ is given by formula (4.14). Again let $Y$ be the number determined by Lemma 1a. Since $B_{m} \neq 0$ we conclude from (4.15) that

$$
B(y)=B_{m} y^{m}[1+o(1)] \text { as }|y| \rightarrow \infty .
$$

This means that $B(y)$ is monotone if $y$ is sufficiently large. We can therefore find a $Y_{0}>Y$ such that $B(y)$ is monotone for $|y| \geqq Y_{0}$. In view of Lemma 1a it is always possible to find a real $y_{0}$ such that $\left|y_{0}\right|>Y_{0}$ and $A_{1}\left(y_{0} \sqrt{\xi_{m}}, y_{0}\right)>0$. Let $\theta$ be an arbitrary real number, then there exists an integer $g$ such that

$$
\theta+2 \pi g \leqq B\left(y_{0}\right)<\theta+2 \pi(g+1) .
$$

We consider from now on only such values of $y$ for which $y y_{0}>0$ and $|y| \geqq y_{0} \quad$ For such values $B(y)$ is either monotone increasing or monotone decreasing. In the first case we can find a value $y_{1}$ such that $B\left(y_{1}\right)=$ $\theta+2(g+1) \pi$; in the second case there exists a value $y_{1}$ for which $B\left(y_{1}\right)=\theta+2 g \pi$. Since $\left|y_{1}\right| \geqq y_{0}$ and $y_{1} y_{0}>0$ we see from Lemma la that $A_{1}\left(y_{1} \sqrt{\xi_{m}}, y\right)>0$ while $B\left(y_{1}\right)-\theta=\beta_{1}\left(y_{1} \sqrt{\xi_{m}}, y_{1}\right)-\theta$ is an integer multiple of $2 \pi$. To complete the proof of Lemma 1 we must only put $y^{*}=y_{1}$ and $t^{*}=y_{1} \sqrt{\xi_{m}}$.

5. Proof of Theorem 1. Let $P(t)=\sum_{v=0}^{m} c_{v} t^{v}$ be a polynomial of degree $m>2,\left(c_{m} \neq 0\right)$ and $\kappa_{n}^{-1}=e_{n}\left(c_{0}\right)$. We carry an indirect proof for 
Theorem 1 and suppose therefore that

$$
f_{n}(t)=\kappa_{n} e_{n}[P(t)]
$$

is a characteristic function. The function $f_{n}(t)$ agrees for real values of $z$ with the function $A(z)=\kappa_{n} e_{n}[P(z)]$ so that the conditions of Theorem $\mathrm{C}$ are satisfied. Hence $f_{n}(t)$ is an entire characteristic function. We consider from now on this characteristic function also for complex values of the argument $z=t+i y$ and can apply Theorem D. The last part of this theorem indicates that necessarily

$$
R(t, y)=\left|\begin{array}{c}
f_{n}(t+i y) \\
f_{n}(i y)
\end{array}\right| \leqq 1
$$

for all real $t$ and $y$.

Define the functions

$$
f_{v}(z)=\kappa_{v} e_{v}[P(z)] \quad(v=1,2, \cdots, n)
$$

where $\kappa_{v}=\left[e_{v}\left(c_{0}\right)\right]^{-1}$ and note that $f_{v}(0)=1 \quad(v=1,2, \cdots, n)$ and that $f_{1}(z)=\exp \left[\phi_{1}(z)\right]$. We (easily) obtain from the definition (5.2) of the functions $f_{v}(z)$ the recursion formula

$$
f_{v}(z)=\exp \left\{\kappa_{v-1}^{-1}\left[f_{v-1}(z)-1\right]\right\} \quad(v=2, \cdots, n) .
$$

We introduce now the functions

$$
\phi_{v}(z)=\kappa_{v-1}^{-1}\left[f_{v-1}(z)-1\right] \quad(v=2,3, \cdots, n)
$$

and write $\alpha_{v}(t, y)$ for the real part and $\beta_{v}(t, y)$ for the imaginary part of $\phi_{v}(z)$ so that

$$
\phi_{v}(z)=\alpha_{v}(t, y)+i \beta_{v}(t, y) \quad(v=1,2, \cdots, n)
$$

and

$$
f_{v}(z)=\exp \left[\phi_{v}(z)\right] \quad(v=1,2, \cdots, n)
$$

Let $\rho_{v}, \lambda_{v}$ be real numbers satisfying

$$
\kappa_{v}^{-1}=\exp \left(\rho_{v}+i \lambda_{v}\right) \quad(v=2,3, \cdots, n) .
$$

Since $\kappa_{v-1}^{-1}=e_{v-1}\left(c_{0}\right)$ we see that $\kappa_{v}^{-1}=\exp \left(\kappa_{v-1}^{-1}\right)$ or $\exp \left(\rho_{v}+i \lambda_{v}\right)=$ $e_{2}\left(\rho_{v-1}+i \lambda_{v-1}\right)$. Therefore

$$
\rho_{v}+i \lambda_{v}=\exp \left(\rho_{v-1}+i \lambda_{v-1}\right)+2 g_{v} \pi i
$$

where $g_{v}$ is an integer. It follows from (5.5.2) that

$$
\lambda_{v}=e^{\rho-1} \sin \lambda_{v-1}+2 g_{v} \pi \text {. }
$$

We combine (5.4.1), (5.4.3) and (5.5.1) to get 
$\phi_{v}(z)=\exp \left(\rho_{v-1}+i \lambda_{v-1}\right)\left\{\exp \left[\alpha_{v-1}(t, y)+i \beta_{v-1}(t, y)\right]-1\right\} \quad(v=2,3, \cdots, n)$.

Separating real an imaginary parts in the last formula we obtain formulae for $\alpha_{v}(t, y)$ and $\beta_{v}(t, y)$

$$
\begin{array}{r}
\alpha_{v}(t, y)=e^{\rho} v_{-1}^{+\alpha_{v-1}(t, y)} \cos \left[\lambda_{v-1}+\beta_{v-1}(t, y)\right]-e^{\rho v-1} \cos \lambda_{v-1} \\
(v=2,3, \cdots, n)
\end{array}
$$

$$
\begin{aligned}
\beta_{v}(t, y)=e^{\rho} v_{-1}^{+\alpha_{v-1}(\ell, y)} \sin \left[\lambda_{v-1}+\beta_{v-1}(t, y)\right]- & e^{\rho v-1} \sin \lambda_{v-1} \\
& (v=2,3, \cdots, n) .
\end{aligned}
$$

Moreover, setting $A_{v}(t, y)=\alpha_{v}(t, y)-\alpha_{v}(0, y)$ we obtain

$$
\begin{aligned}
& A_{v}(t, y)=\left\{e^{A} v_{-1}^{(t, y)} \cos \left[\lambda_{v-1}+\beta_{v-1}(t, y)\right]-\cos \left[\lambda_{v-1}+\beta_{v-1}(0, y)\right]\right\} \\
& \quad \times \exp \left[\rho_{v-1}+\alpha_{v-1}(0, y)\right] \\
& (v=2,3, \cdots, n) .
\end{aligned}
$$

We apply now Lemma 1 and select $\theta=-\lambda_{1}$. Then it is possible to find a pair of real numbers $t^{*}, y^{*}$ such that

$$
A_{1}\left(t^{*}, y^{*}\right)>0
$$

while

$$
\beta_{1}\left(t^{*}, y^{*}\right)+\lambda_{1}=2 g_{1} \pi
$$

( $g_{1}$ an integer) .

We show next by induction that a similar relation holds for all functions $\beta_{v}(t, y)$ namely that

$$
\beta_{v}\left(t^{*}, y^{*}\right)+\lambda_{v}=2 g_{v} \pi
$$

Here $t^{*}, y^{*}$ are the values determined by Lemma $1, \lambda_{v}$ is given by (5.5.3) and $g_{v}$ is an integer. We prove (5.9) by induction. Formula (5.8.2) indicates that (5.9) is valid for $v=1$, we suppose now that it holds for all subscripts inferior to $v$. In particular then, $\beta_{v-1}\left(t^{*}, y^{*}\right)+\lambda_{v-1}=$ $2 g_{v-1} \pi$. Substituting this into (5.6.2) we see that $\beta_{v}\left(t^{*}, y^{*}\right)=-e^{\rho_{v-1}} \sin \lambda_{v-1}=$ $-\lambda_{v}+2 g_{v} \pi$. Thus (5.9) is generally valid.

We see from (5.7) and (5.9) that

$$
\begin{aligned}
& A_{v}\left(t^{*}, y^{*}\right)=\left\{e^{A} v_{-1}\left(t^{*}, y^{*}\right)-\cos \left[\lambda_{v-1}+\beta_{v-1}\left(0, y^{*}\right)\right]\right\} \exp \left[\rho_{v-1}+\alpha_{v-1}\left(0, y^{*}\right)\right] \\
& (v=2,3, \cdots, n) \text {. }
\end{aligned}
$$

From this formula we see that the relation $A_{v-1}\left(t^{*}, y^{*}\right)>0$ implies $A_{v}\left(t^{*}, y^{*}\right)>0$. We can therefore conclude from (5.8.1) that

$$
A_{n}\left(t^{*}, y^{*}\right)>0 \text {. }
$$

It follows immediately from (5.1), (5.4.2) and (5.4.3) that $R(t, y)=$ $\exp \left\{A_{n}(t, y)\right\}$. We have therefore determined values $t^{*}, y^{*}$ such that

$$
R\left(t^{*}, y^{*}\right)>1 \text {. }
$$


But this contradicts (5.1) which must be satisfied if $f_{n}(t)$ is a characteristic function. This contradiction completes the proof of Theorem 1 since it shows that $f_{n}(t)$ can not be a characteristic function if $m>2$.

In case $m \geqq 2$ the iterated exponential polynomials $f_{n}(t)=e_{n}[P(t)]$ can be characteristic functions. The function $f_{1}(t)=\exp \left(-a_{2} t^{2}+i a_{1} t\right)$ where $a_{1}$ and $a_{2}$ are both real, $a_{2} \geqq 0$, is a characteristic function; it follows then from the recursion formula (5.3) and a Theorem of B. de Finetti [4] that $f_{v}^{\prime}(z)$ as defined by (5.2) is a characteristic function for all values of $v$. For the sake of completeness we quote de Finetti's result.

Theorem of de Finetti. If $f(t)$ is an arbitrary characteristic function and if $p$ is a positive real number then $g(t)=\exp \{p[f(t)-1]\}$ is also a characteristic function.

6. Proof of Theorem 2. In this section we investigate the function

$$
f(t)=\exp \left[\lambda_{1}\left(e^{i t}-1\right)+\lambda_{2}\left(e^{-i t}-1\right)+P_{m}(t)\right]
$$

where

$$
P_{m}(t)=\sum_{v=0}^{m} c_{v} t^{v}\left(c_{m} \neq 0\right)
$$

is a polynomial of degree $m$.

If $f(t)$ is a characteristic function then it must be, according to Theorem $\mathrm{C}$, an entire characteristic function and we write

$$
f(z)=\exp \left\{\lambda_{1}\left(e^{i z}-1\right)+\lambda_{2}\left(e^{-i z}-1\right)+P_{m}(z)\right\}
$$

where $z=t+i y$. We can apply Theorem $\mathrm{D}$ and see that necessarily

$$
R(t, y)=\left|\begin{array}{c}
f(t+i y) \\
f(i y)
\end{array}\right| \leqq 1
$$

for all real $t$ and $y$.

The familiar normalization of characteristic function $[f(0)=1]$ indicates that it is no restriction to assume that $c_{0}=0$. We write

$$
\begin{aligned}
& f_{1}(z)=\exp \left[\lambda_{1}\left(e^{i z}-1\right)+\lambda_{2}\left(e^{-i z}-1\right)\right], \\
& f_{2}(z)=\exp \left[\sum_{v=1}^{m} c_{v} z^{v}\right]
\end{aligned}
$$

so that $f(z)=f_{1}(z) f_{2}(z)$.

We see easily that 


$$
\left|\frac{f_{1}(t+i y)}{f_{1}(i y)}\right|=\exp \left[(1-\cos t)\left(-\lambda_{1} e^{-y}-\lambda_{2} e^{y}\right)\right] .
$$

Since $f_{2}(z)=\exp \left[P_{m}(t)-c_{0}\right]=\exp \left[\alpha_{1}(t, y)+i \beta_{1}(t, y)\right]$ we get, using the notation of Lemma 1 ,

$$
\left|\frac{f_{2}(t+i y)}{f_{2}(i y)}\right|=\exp \left[A_{1}(t, y)\right] \text {. }
$$

From (6.1), (6.2), (6.3.1) and (6.3.2) we obtain

$$
R(t, y)=\exp \left[(1-\cos t)\left(-\lambda_{1} e^{-y}-\lambda_{2} e^{y}\right)+A_{1}(t, y)\right] .
$$

We show first by means of an indirect proof that $m<3$. Suppose therefore that $m \geqq 3$, we can then apply Lemma 1 a and see that there exist $\xi_{m} \geqq 0$ and $y$ such that $A_{1}\left(y \sqrt{\xi_{m}}, y\right)>0$ provided that $y$ satisfies either condition (i) or condition (ii) of Lemma 1a. Substituting $t=y \sqrt{\bar{\xi}_{m}}$ into (6.4) we get

$$
R\left(y \sqrt{\xi_{m}}, y\right)=\exp \left[\left(1-\cos y \sqrt{\xi_{m}}\right)\left(-\lambda_{1} e^{-y}-\lambda_{2} e^{y}\right)+A_{1}\left(y \sqrt{\xi_{m}}, y\right)\right]
$$

We select now an integer $k$ which satisfies one of the following two conditions :

(a) if $m>3$ or if $m=3$ but $\beta_{3}=0$ then $k>\left(Y \sqrt{\xi_{m}}\right) /(2 \pi)$

(b) if $m=3$ and $\beta_{3} \neq 0$ then $\left(-\operatorname{sign} \beta_{3}\right) k>\left(Y \sqrt{\xi_{m}}\right) /(2 \pi)$

Then $y=\frac{2 \pi k}{\sqrt{\xi_{m}}}$ satisfies either condition (i) or condition (ii) of Lemma 1a. We substitute in the preceding formula $y=(2 \pi k) / \sqrt{\xi_{m}}$ and obtain

$$
R\left(2 \pi k, \frac{2 \pi k}{\sqrt{\xi_{m}}}\right)=\exp \left[A_{1}\left(2 \pi k, \frac{2 \pi k}{\sqrt{\xi_{m}}}\right)\right]>1
$$

in contradiction with (6.2). Thus we have proven that necessarily $m \leqq 2$.

In this case we have

$$
A_{1}(t, y)=\alpha_{1} t+\alpha_{2} t^{2}-2 \beta_{2} t y
$$

and

(6.5) $R(t, y)=\exp \left[(1-\cos t)\left(-\lambda_{1} e^{-y}-\lambda_{2} e^{y}\right)+\alpha_{1} t+\alpha_{2} t^{2}-2 \beta_{2} t y\right]$.

We prove next that $\lambda_{1}$ and $\lambda_{2}$ are non-negative. If either $\lambda_{1}$ or $\lambda_{2}$ is negative we choose $t=\pi$, then

$$
R(\pi, y)=\exp \left[2\left(-\lambda_{1} e^{-y}-\lambda_{2} e^{y}\right)+\alpha_{1} \pi+\alpha_{2} \pi^{2}-2 \beta_{2} \pi y\right] .
$$

It is then possible to make the exponent on the right side of (6.5) positive 
by selecting $y$ sufficiently large and giving it an appropriate sign. Therefore there exists a value $y^{*}$ such that $R\left(\pi, y^{*}\right)>1$ in contradiction with (6.2). Morever it follows from the hermitian property $[f(-t)=$ $\overline{f(t)]}$ of characteristic funcions that $c_{1}$ is purely imaginary while $c_{2}$ is real. We conclude finally from the boundedness of characteristic functions that $c_{2}$ is negative. Writing $c_{1}=i \alpha_{1}, c_{2}=-a_{2}$ we obtain the complete statement of Theorem 2.

Theorem $\mathrm{B}$ is also a special case of Theorem 2 ; it is obtained by putting $\lambda_{1}=\lambda_{2}=0$.

7. Proof of Theorem A. In this section ${ }^{3}$ we consider an entire characteristic function $f(z)$ of finite order $\rho$. By Hadamard's factorization theorem we can write $f(z)$ in the form

$$
f(z)=G(z) e^{H(z)}
$$

where $G(z)$ is the canonical product of the zeros of $f(z)$ and $H(z)$ is a polynomial of degree $m \leqq \rho$. We denote by $\rho_{1}$ the exponent of convergence of the zeros of $f(z)$. It is easily seen that $\rho=\max \left(\rho_{1}, m\right)$. If $\rho_{1}<\rho$ then necessarily $\rho=m$. It is known that the order of a canonical product equals its exponent of convergence. Let $G(z)$ be a canonical product of order $\rho_{1}$, then for any $\varepsilon>0$ the modulus $|G(z)| \leqq$ $\exp \left(|z|^{\rho_{1}+\varepsilon}\right)$ provided that $|z|$ is sufficiently large. We will also use the following result which is due to E. Borel :

If $G(z)$ is a canonical product of order $\rho_{1}$ and if $\varepsilon$ is an arbitrary positive number then there exists an infinite number of circles of arbitrarily large radius on which the inequality

$$
|G(z)|>\exp \left(-|z|^{p_{1}+\varepsilon}\right)
$$

holds.

Let $z=t+i y$ and denote by $r=|z|=\sqrt{t^{2}+y^{2}}$. We see then that there exist arbitrarily large values of $r$ such that

$$
|G(t+i y)|>\exp \left(-r^{\rho_{1}+\varepsilon}\right) .
$$

On the other hand, we know that for arbitrary $\varepsilon>0$ and sufficiently large $y$

$$
|G(i y)| \leqq \exp \left(y^{\rho_{1}+\varepsilon}\right) \leqq \exp \left(r^{\rho_{1}+\varepsilon}\right) .
$$

We combine the last two inequalities and see that there exists an increasing sequence $\left\{r_{k}\right\}$ of positive real number such that $\lim _{k \rightarrow \infty} r_{k}=\infty$ and that for arbitrary $\varepsilon>0$ and sufficiently large $k$

${ }^{3} \mathrm{We}$ use in the following certain theorems from the theory of functions of a complex variable. The needed results may be found, for instance, in [2], pp. 165-175. 


$$
R_{1}(t, y)=\left|\begin{array}{c}
G(t+i y) \\
G(i y)
\end{array}\right|>\exp \left(-2 r_{k}^{\rho_{1}+\varepsilon}\right)
$$

provided that $\sqrt{t^{2}+y^{2}}=r_{k}$.

We consider next $f_{2}(z)=e^{H(z)}$ and write

$$
R_{2}(t, y)=|\exp [H(t+i y)-H(i y)]|
$$

so that

$$
R(t, y)=\left|\frac{f(t+i y)}{f(i y)}\right|=R_{1}(t, y) R_{2}(t, y)
$$

We give an indirect proof for the statement of Theorem A and assume therefore that $f(z)$ is an entire characteristic function of order $\rho>2$ and suppose that the exponent of convergence $\rho_{1}$ of the zeros of $f(z)$ is less than $\rho ; \rho_{1}<\rho$. Then necessarily (Theorem D)

$$
R(t, y) \leqq 1
$$

for all real $t$ and $y$.

Since $\rho_{1}<\rho$ we have necessarily $\rho=m$, the degree of the polynomial $H(z)$. As a characteristic function $f(z)$ must satisfy the condition $f(0)=1$ so that $H(0)=0$. We can then use the notation of Lemma 1 and write

$$
\phi_{1}(z)=H(z)=\sum_{v=1}^{m}\left(\alpha_{v}+i \beta_{v}\right) z^{y}
$$

so that

$$
R_{2}(t, y)=\exp \left[A_{1}(t, y)\right] .
$$

We see then from (7.2), (7.4) and (7.6) that there exists an infinite sequence $\left\{r_{k}\right\}$ of indefinitely increasing real numbers such that for an arbitrary $\varepsilon>0$

$$
R(t, y)>\exp \left[-2 r_{k}^{\rho_{1}+\varepsilon}+A_{1}(t, y)\right]
$$

provided that $k$ is sufficiently large and that $t^{2}+y^{2}=r_{k}^{2}$.

We define now an infinite sequence of points $\left(t_{k}, y_{k}\right)$ in the $z$-plane. In order to be able to apply Lemma 1a we subject these points to the following restrictions :

( i ) $t_{k}=y_{k} \sqrt{\xi_{m}}$

(ii ) $\left|t_{k}+i y_{k}\right|=r_{k}$

(iii) if $m>3$ or $m=3$ while $\beta_{3}=0$ then $y_{k}>0$

(iv) if $m=3$ and $\beta_{3} \neq 0$ then $\left(-\operatorname{sign} \beta_{3}\right) y_{k}>0$ 
From (i) and (ii) we see that all these points are located in the same quadrant and that $\left|y_{k}\right|=r_{k} / V 1+\bar{\xi}_{m}$. We deduce from Lemma 1a that

$$
A_{1}\left(t_{k}, y_{k}\right)=\tilde{A}\left|y_{k}\right|^{m}[1+o(1)] \text { as } k \rightarrow \infty \text {. }
$$

We denote by $C=A\left(1+\xi_{m}\right)^{-m / 2}$ and obtain from (7.7) and (7.8)

$$
R\left(t_{k}, y_{k}\right)>\exp \left\{-2 r_{k}^{\rho_{1}+\varepsilon}+C r_{k}^{m}[1+o(1)]\right\} \text { as } k \rightarrow \infty .
$$

Since by assumption $\rho=m>\rho_{1}$, we can choose the arbitrary positive quantity $\varepsilon$ so that $\rho_{1}+\varepsilon<m$; we conclude then from the last inequality that

$$
R\left(t_{k}, y_{k}\right)>\exp \left\{C r_{k}^{m}[1+o(1)]\right\} \text { as } k \rightarrow \infty .
$$

Since $C>0$ we can determine $k$ so large that $R\left(t_{k}, y_{k}\right)>1$. This, however, contradicts (7.5) and we see therefore that $f(z)$ can not be a characteristic functions and have therefore completed the proof of Theorem A.

\section{REFERENCES}

1. R. P. Boas, Sur les séries et integrales de Fourier à coefflcients positives, C. R. Acad. Sci. Paris 225, (1949), 1837-1838.

2. E. T. Copson, Theory of functions of a complex variable, Oxford, 1955, Clarendon Press.

3. D. Dugué, Analycité et convexité des founctions caractéristiques, Ann. Inst. H. Poincaré 12 (1951), 45-46.

4. B. de Finetti, Le funzione caracteristiche di legge instantanea, Rend. R. Acc. Naz. Lincei ser 6, 12, (1930), 278-282.

5. G. Kunetz, Sur quelques propriétés des fonctions caractéristiques, C. R. Acad. Sci. Paris, (1936), 1829-1832.

6. G. Kunetz, Sur quelques propriétés des fonctions caractéristiques, Librairie L. Rodstein, Paris, (1937).

7. M. Loève, Probability theory, D. Van Nostrand Company, New York, 1955.

8. E. Lukacs O. Szász, On analytic characteristic functions, Pacific J. Math. 2 (1952), 615-625.

9. E. Lukacs, Les fonctions caractéristiques analytiques, 15 (1957), 217-251 Ann. Inst. H. Poincaré.

10. J. Marcinkiewicz, Sur une propriété de la loi de Gauss, Math. Z. 44, (1938) 612-618.

The Catholic University of America 



\section{PACIFIC JOURNAL OF MATHEMATICS}

\section{EDITORS}

David Gilbarg

Stanford University

Stanford, California

R. A. Beaumont

University of Washington

Seattle 5, Washington

\author{
A. L. Whiteman
}

University of Southern California

Los Angeles 7, California

E. G. Straus

University of California

Los Angeles 24, California

\section{ASSOCIATE EDITORS}

\author{
E. F. BECKENBACH \\ C. E. BURGESS \\ M. HALL \\ E. HEWITT
}

\author{
A. HORN \\ V. GANAPATHY IYER \\ R. D. JAMES \\ M. S. KNEBELMAN
}

L. NACHBIN
I. NIVEN
T. G. OSTROM
H. L. ROYDEN

M. M. SCHIFFER

G. SZEKERES

F. WOLF

K. YOSIDA

\section{SUPPORTING INSTITUTIONS}

\author{
UNIVERSITY OF BRITISH COLUMBIA \\ CALIFORNIA INSTITUTE OF TECHNOLOGY \\ UNIVERSITY OF CALIFORNIA \\ MONTANA STATE UNIVERSITY \\ UNIVERSITY OF NEVADA \\ OREGON STATE COLLEGE \\ UNIVERSITY OF OREGON \\ UNIVERSITY OF SOUTHERN CALIFORNIA
}

\author{
STANFORD UNIVERSITY \\ UNIVERSITY OF UTAH \\ WASHINGTON STATE COLLEGE \\ UNIVERSITY OF WASHINGTON \\ AMERICAN MATHEMATICAL SOCIETY \\ CALIFORNIA RESEARCH CORPORATION \\ HUGHES AIRCRAFT COMPANY \\ THE RAMO-WOOLDRIDGE CORPORATION
}

Mathematical papers intended for publication in the Pacific Journal of Mathematics should be typewritten (double spaced), and the author should keep a complete copy. Manuscripts may be sent to any of the editors. All other communications to the editors should be addressed to the managing editor, E. G. Straus at the University of California, Los Angeles 24, California.

50 reprints per author of each article are furnished free of charge; additional copies may be obtained at cost in multiples of 50 .

The Pacific Journal of Mathematics is published quarterly, in March, June, September, and December. The price per volume (4 numbers) is $\$ 12.00$; single issues, $\$ 3.50$. Back numbers are available. Special price to individual faculty members of supporting institutions and to individual members of the American Mathematical Society: $\$ 4.00$ per volume; single issues, $\$ 1.25$.

Subscriptions, orders for back numbers, and changes of address should be sent to Pacific Journal of Mathematics, 2120 Oxford Street, Berkeley 4, California.

Printed at Kokusai Bunken Insatsusha (International Academic Printing Co., Ltd.), No. 6, 2-chome, Fujimi-cho, Chiyoda-ku, Tokyo, Japan.

\section{PUBLISHED BY PACIFIC JOURNAL OF MATHEMATICS, A NON-PROFIT CORPORATION}

The Supporting Institutions listed above contribute to the cost of publication of this Journal, but they are not owners or publishers and have no responsibility for its content or policies. 


\section{Pacific Journal of Mathematics}

\section{Vol. 8, No. 3 \\ May, 1958}

Michael Israel Aissen, A set function defined for convex plane domaines... . 383

Robert Ellis, Distal transformation groups ................... 401

Ciprian Foias, On a commutative extension of a commutative Banach algebra ....................................... 407

Jerry William Gaddum, Linear inequalities and quadratic forms ......... 411

Allen A. Goldstein and Elliott Ward Cheney, Jr., A finite algorithm for the solution of consistent linear equations and inequalities and for the Tchebycheff approximation of inconsistent linear equations...........

William L. Hart and T. S. Motzkin, Proof of the fundamental theorem on implicit functions by use of composite gradient corrections .......... 429

Henry Berge Helson, Conjugate series and a theorem of Paley .......... 437

Wu-Chung Hsiang, Abelian groups characterized by their independent subsets....................................... 447

John W. Lamperti, On the isometries of certain function-spaces ........ 459

Karel DeLeeuw and Walter Rudin, Extreme points and extremum problems

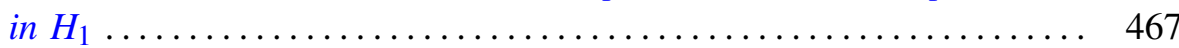

Eugene Lukacs, Some extensions of a theorem of Marcinkiewicz........ 487

George W. Mackey, Multiplicity free representations of finite groups ..... 503

Eben Matlis, Injective modules over Noetherian rings ............. 511

John William Neuberger, Continuous products and nonlinear integral equations

Lawrence Edward Payne and Hans F. Weinberger, New bounds for solutions of second order elliptic partial differential equations...

William T. Reid, A Prüfer transformation for differential systems ........ 575

Howard L. Rolf, The free lattice generated by a set of chains ...

K. M. Saksena, Inversion and representation theorems for a generalized

Laplace integral....................................... 597

Daniel Shanks, Two theorems of Gauss......................... 609

Paul Slepian, On the Lebesgue area of a doubled map ............... 613

Otto Szász and Nelson Paul Yeardley, Jr., The representation of an analytic function by general Laguerre series . ..................... 621

Alan C. Woods, On two-dimensional convex bodies ................. 635 